\title{
Avaliação do comportamento eletrocinético de suspensões cerâmicas não-aquosas contendo PVB como aditivo
}

\author{
(Evaluation of the electrokinetic behavior of non-aqueous ceramic \\ suspensions containing PVB)
}

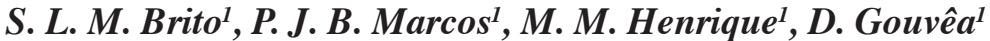 \\ ${ }^{1}$ Laboratório de Processos Cerâmicos - Departamento de Engenharia Metalúrgica e de Materiais \\ Escola Politécnica da Universidade de S. Paulo (EPUSP) \\ Av. Prof Mello Moraes, 2463, Cidade Universitária, S. Paulo 05580-900 \\ dgouvea@usp.br
}

\begin{abstract}
Resumo
Produtos cerâmicos à base de alumina $\left(\mathrm{Al}_{2} \mathrm{O}_{3}\right)$, zircônia $\left(\mathrm{ZrO}_{2}\right)$ e/ou carbeto de silício $(\mathrm{SiC})$ têm grande utilização em várias áreas e com diferentes formas que vão desde corpos sinterizados até pós abrasivos. As etapas fundamentais do processamento para a fabricação de cerâmicas são sua mistura e conformação. A conformação cerâmica pode ser feita por vários métodos e o sucesso da utilização destas técnicas depende fundamentalmente do conhecimento das propriedades eletrocinéticas da dispersão de partículas com seus aditivos. Este trabalho teve como objetivo o estudo das características eletrocinéticas de dispersões de $\mathrm{Al}_{2} \mathrm{O}_{3}, \mathrm{ZrO}_{2}$ ou $\mathrm{SiC}_{1}$ em etanol, analisando a formação de cargas devido à adsorção específica de aditivos (como íons $\mathrm{Al}^{3+}$ para o caso da $\mathrm{Al}_{2} \mathrm{O}_{3}$ ou $\mathrm{SiC}_{\text {) }} \mathrm{e}$ a influência de outro aditivo como polivinil butiral (PVB) na estabilidade da dispersão. Podemos constatar que para os três sistemas ocorre adsorção do PVB, e que o mesmo adsorve preferencialmente quando a superfície da partícula está carregada positivamente, situação que para o $\mathrm{ZrO}_{2}-\mathrm{MgO}$ ocorre espontaneamente quando adicionado em etanol, já para o caso do $\mathrm{Al}_{2} \mathrm{O}_{3}$ e $\mathrm{SiC}_{\text {consegue-se }}$ adicionando uma solução alcóolica de $\mathrm{Al}\left(\mathrm{NO}_{3}\right)_{3}$ promovendo adsorção de íons $\mathrm{Al}^{3+}$.

Palavras-chave: suspensão cerâmica, alumina, zircônia, carbeto de silício, PVB, adsorção, comportamento eletrocinético.
\end{abstract}

\begin{abstract}
Alumina $\left(\mathrm{Al}_{2} \mathrm{O}_{3}\right)$, zirconia $\left(\mathrm{ZrO}_{2}\right)$ and silicon carbide ( $\left.\mathrm{SiC}\right)$ based ceramics have a large number of applications in the ceramic industry. Among the most relevant stages of the fabrication process of these materials, mixture and shaping deserve special attention. The success of these stages depends on the knowledgement of the suspension electrokinetics properties containing the appropriate additives. In this work, the electrokinetics properties of ethanolic suspensions of $\mathrm{Al}_{2} \mathrm{O}_{3}, \mathrm{ZrO}$ or $\mathrm{SiC}$ were studied as a function of the adsorption of specific additives. Al ${ }^{3+}$ was tested on $\mathrm{Al}_{2} \mathrm{O}_{3}$ and $\mathrm{SiC}$ suspensions, and polyvinylbutyral (PVB) was tested on all suspensions to evaluate its influence on the suspension stability. It was shown that PVB is adsorbed on the surface of all powders under experiment, and its adsorption was observed to be higher when the surface was positively charged. These surface positive charges were spontaneously generated on $\mathrm{ZrO}_{2}-\mathrm{MgO}$ ethanol suspension. However on $\mathrm{Al}_{2} \mathrm{O}_{3}$ and SiC, this surface situation was only achieved after the adsorption of $\mathrm{Al}^{3+}$.
\end{abstract}

Keywords: ceramic suspensions, alumina, zirconia, silicon carbide, PVB, electrokinetic behavior.

\section{INTRODUÇÃO}

A conformação é uma das etapas mais importantes da produção de materiais cerâmicos e envolve principalmente conceitos da química coloidal, empacotamento de partículas e de reologia de materiais [1-7]. Estes processos consistem em diferentes metodologias que visam formar corpos sólidos com formas definidas.

Os métodos de conformação são determinados pelas características das matérias-primas, pela sua concentração e pela forma final do produto desejado [8]. A grande maioria de tais metodologias empregadas industrialmente utiliza uma solução aquosa ou orgânica como meio fluido criando uma suspensão de partículas, com maior ou menor concentração de sólidos. Dentre as metodologias que utilizam suspensões fluídas de baixa viscosidade para a conformação de corpos cerâmicos pode-se citar a colagem de barbotina (slip casting) [9,10], deposição eletroforética (EPD) $[11,12]$ e a conformação por gel (gel casting) [13, 14]. As suspensões são dependentes tanto da fase dispersante 
como da fase dispersa, ou seja, respectivamente do meio fluido e de todos os elementos que se encontram dispersos ou solúveis nele tais como partículas sólidas, dispersantes, ligantes, plastificantes ou outros aditivos, os quais possuem finalidades específicas.

Dentre as várias metodologias possíveis para a conformação de materiais cerâmicos a partir de soluções encontra-se a técnica de deposição eletroforética, a qual tem recebido uma atenção particularmente crescente nos últimos anos devido à sua versatilidade, facilidade de controle e baixo custo de produção $[15,16]$. A metodologia contempla tanto a utilização de solventes aquosos como de não-aquosos, sendo que os primeiros possuem a limitação da eletrólise e os últimos são muito criticados por: - 1 apresentarem baixos valores de carga elétrica superficial (essencial para os processos de estabilização eletrostática e eletroestérica), - 2 elevada volatilidade e - 3 riscos à saúde e ao meio ambiente [17]. No entanto, é possível utilizar alguns solventes orgânicos, de baixa toxidade, volatilidade e que não apresentam sérios riscos ao meio ambiente e à saúde humana [18-20].

É conhecido que os solventes não-aquosos, de modo geral, apresentam baixas constantes dielétricas, quando comparados à água (para a $\mathrm{H}_{2} \mathrm{O}, \varepsilon=78$ ) [21]. No entanto, estudos realizados em suspensões não-aquosas de alguns óxidos cerâmicos comprovam que determinadas reações superficiais entre os sólidos e o solvente podem gerar cargas elétricas cujas magnitudes são adequadas ao processo de estabilização eletrocinética das mesmas [15, 18, 22-24]. Tais magnitudes chegam a ser superiores às magnitudes apresentadas pelos mesmos materiais quando em meios aquosos, o que justifica o emprego de tais solventes.

Tendo em vista o processo de conformação e a posterior etapa de secagem, para a eliminação do solvente orgânico, faz-se necessária à utilização de aditivos que confiram características de resistência mecânica. No caso, tais aditivos devem ser compatíveis com o solvente e com o tipo de sólido utilizado. A contribuição das cargas geradas, assim como da adsorção de aditivos na superfície dos materiais sólidos, no processo de estabilização de suspensões pode ser avaliada por técnicas eletrocinéticas, visto que estas dependem

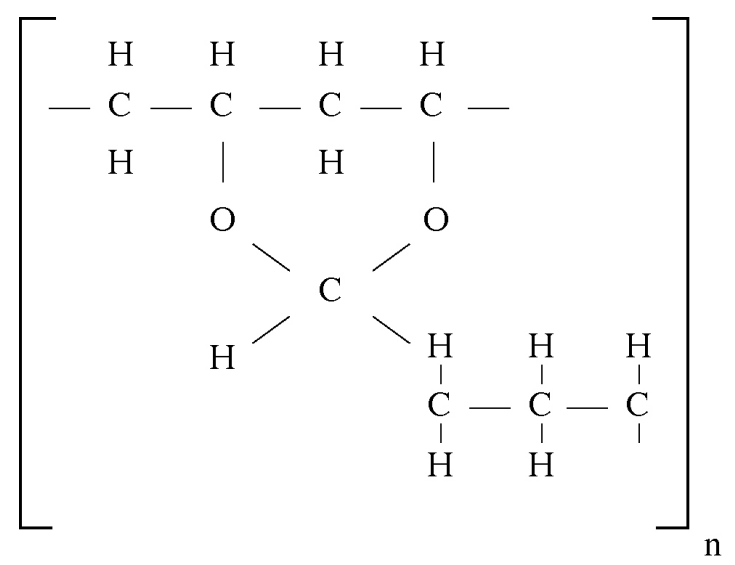

Figura 1: Estrutura molecular do poli(vinil butiral).

[Figure 1: Molecular structure of poly(vinyl butyral).] exatamente da presença de cargas elétricas superficiais [25]. Um dos aditivos escolhidos para este trabalho foi o poli(vinil butiral), PVB, que é muito empregado em processamento cerâmico como ligante e conhecido pelas suas características adesivas em superfícies vítreas [24].

A molécula de PVB é considerada como sendo um polímero de peso molecular médio $(30.000 \mathrm{~g} / \mathrm{mol})$ e que apresenta grupos funcionais não-iônicos, de acordo com a estrutura ilustrada na Fig. 1:

A sua principal vantagem é que ao ser devidamente plastificado pelo solvente confere elevada resistência mecânica ao corpo sólido conformado, além de ser facilmente pirolisado.

Neste trabalho serão apresentados os resultados da avaliação de eficiência de estabilização de suspensões nãoaquosas de três dos materiais cerâmicos mais amplamente utilizados pela indústria $\left(\mathrm{Al}_{2} \mathrm{O}_{3}, \mathrm{SiC}\right.$ e $\left.\mathrm{ZrO}_{2}-\mathrm{MgO}\right)$ através de caracterização eletrocinética. A diferença de eficiência na estabilização gerada pela mudança de $\mathrm{pH}$ e adsorção específica de íons, além da adsorção de moléculas poliméricas (PVB) serão discutidas.

\section{MATERIAIS E MÉTODOS}

\section{Matérias-primas}

Os pós utilizados neste trabalho foram fornecidos pela Alcoa Alumínio S. A. $\left(\mathrm{Al}_{2} \mathrm{O}_{3}\right.$ e $\left.\mathrm{SiC}\right)$ e pela Usina Piloto de produção experimental de $\mathrm{ZrO}_{2}$ do IPEN (Instituto de Pesquisas Energéticas e Nucleares). As características dos pós foram obtidas através de análises de área específica por adsorção física de $\mathrm{N}_{2}$ (Gemini 2375 Micromeritics - pré-tratamento a $200{ }^{\circ} \mathrm{C}$ e $100 \mu \mathrm{m}$ de $\mathrm{Hg}$ - método BET) e distribuição de tamanho de partículas por espalhamento laser (Malvern série 2600). Ao $\mathrm{ZrO}_{2}$ foi adicionado $\mathrm{MgO}$ na proporção de $3 \%$ em peso $(8,6 \% \mathrm{em} \mathrm{mol})$, no intuito de promover a estabilização de fases cristalinas no material após futuro tratamento térmico [26-28].

\section{Suspensões cerâmicas}

As suspensões foram preparadas a partir da dispersão das partículas cerâmicas em etanol $\left(\mathrm{C}_{2} \mathrm{H}_{5} \mathrm{OH}\right.$ Absoluto - Synth), com concentrações de 5 e $3 \%$ em volume para o $\mathrm{Al}_{2} \mathrm{O}_{3} \mathrm{e}$ o $\mathrm{SiC}$, respectivamente, e numa concentração de $2 \%$ em volume para o $\mathrm{ZrO}_{2}-\mathrm{MgO}$. Antes das análises, as suspensões foram submetidas à agitação ultra-sônica por 5 min para homogeneização e quebra de possíveis aglomerados.

Com base na literatura [29], podemos observar que o $\mathrm{Al}\left(\mathrm{NO}_{3}\right)_{3}$ é uma das substâncias com capacidade de fornecer íons determinantes de potencial de superfície em meios orgânicos, gerando cargas superficiais suficientes para dispersão do material particulado em alguns casos. Para as suspensões de $\mathrm{Al}_{2} \mathrm{O}_{3}$ e de $\mathrm{SiC}$ foi preparada uma solução alcoólica de nitrato de alumínio $\left(\mathrm{Al}\left(\mathrm{NO}_{3}\right)_{3} \cdot 9 \mathrm{H}_{2} \mathrm{O}\right.$ - numa concentração de $\left.0,5 \mathrm{M}, \mathrm{pa}_{\mathrm{H}}=1,9\right)$. O objetivo desta solução é controlar a estabilidade do sistema através da geração 
de cargas superficiais nas partículas, a partir da adsorção específica do íon $\mathrm{Al}^{3+}$, ou seja, a estabilidade das suspensões ocorrendo por mecanismos eletrostáticos.

A medida de $\mathrm{pH}$ em soluções não-aquosas $\left(\mathrm{pa}_{\mathrm{H}}\right)$ foi realizada com base na correção proposta por Sarkar e Nicholson [15, 18], que relaciona o valor medido experimentalmente $\left(\mathrm{pH}_{\text {exp }}\right)$ num eletrodo para soluções aquosas em meios não-aquosos:

$$
\mathrm{pa}_{\mathrm{H}}=\mathrm{pH}_{\exp }+1,3
$$

Para as suspensões de $\mathrm{Al}_{2} \mathrm{O}_{3}$ e de $\mathrm{SiC}$, o aditivo PVB adicionado, foi preparado pela solubilização de $40 \mathrm{~g}$ do material em $1 \mathrm{~L}$ de etanol. Nas suspensões contendo $\mathrm{ZrO}_{2}-\mathrm{MgO}$ foi empregada uma solução alcoólica de PVB na concentração de $30 \mathrm{~g} / \mathrm{L}$.

No intuito de se constatarem possíveis variações na densidade e viscosidade das suspensões pela adição do PVB foram realizadas medidas de densidade através de um picnômetro e de viscosidade em um viscosímetro de Ostwald. Neste último, a medida de viscosidade está baseada no fluxo de solução através de um tubo capilar. A pressão sob cuja ação o líquido passa a fluir fornece o esforço de cisalhamento [30].

A determinação das características da interface partículalíquido em suspensões pode ser realizada por métodos eletroacústicos desde que existam cargas elétricas presentes na superfície das partículas. Quando estas partículas carregadas são submetidas a um campo elétrico alternado (efeito ESA), executam um movimento oscilatório na mesma freqüência e, devido à diferença de densidade existente entre o sólido e o solvente surge uma transferência de energia na forma de onda sonora para a solução, a qual é detectada por um sensor piezelétrico [31].

A energia acústica gerada é proporcional à mobilidade dinâmica das partículas. A relação entre a mobilidade dinâmica e o sinal acústico medido na suspensão é dado pela equação B [32]:

$$
\mu_{\mathrm{d}}=\mathrm{ESA} /(\phi \cdot \Delta \rho \cdot \mathrm{c})
$$

onde $\mu_{d}$ é a mobilidade dinâmica, ESA a amplitude sônica eletrocinética, $\phi$ a fração volumétrica de sólidos dispersos no solvente, $\Delta \rho$ a diferença de densidade entre a partícula e o solvente, e c a velocidade do som no solvente.

O equipamento utilizado para as análises de mobilidade dinâmica das suspensões foi ESA-8000 (Matec Applied Sciences ${ }^{\mathrm{TM}}$ ). Ele é composto por uma unidade de geração de radiofreqüência, uma célula de medida (contendo as sondas eletroacústica, de $\mathrm{pH}$, de condutividade e de temperatura) envolta por uma jaqueta acrílica externa para o controle de temperatura através de circulação de água, uma bureta digital (para titulações potenciométricas e adição controlada de aditivos) e interface para computador. As medidas de mobilidade dinâmica $\left(\mu_{\mathrm{d}}\right)$ foram realizadas em função da adição de soluções alcoólicas de $\mathrm{HNO}_{3}-2 \mathrm{~N}$ (Synth), de $\mathrm{Al}\left(\mathrm{NO}_{3}\right)_{3}$ (Synth, 99,4\%) 0,5M de HCl-2N
(Synth, 38\%) e de PVB a 30 e $40 \mathrm{~g} / \mathrm{L}$.

\section{RESULTADOS E DISCUSSÃO}

\section{Matérias-primas}

As características dos pós de $\mathrm{Al}_{2} \mathrm{O}_{3}, \mathrm{SiC}$ e $\mathrm{ZrO}_{2}-\mathrm{MgO}$ avaliadas por adsorção física de $\mathrm{N}_{2}$ e espalhamento de luz laser são apresentadas na Tabela I.

Observa-se que o elevado valor de área de superfície

Tabela I - Características gerais das matérias-primas. [Table I - General features of raw materials.]

\begin{tabular}{ccc}
\hline Material & $\mathrm{S}_{\mathrm{BET}}\left(\mathrm{m}^{2} / \mathrm{g}\right)$ & $\mathrm{D}_{50}(\mu \mathrm{m})$ \\
\hline $\mathrm{Al}_{2} \mathrm{O}_{3}$ & 8,4 & 0,4 \\
$\mathrm{SiC}$ & 3,5 & 9,0 \\
$\mathrm{ZrO}_{2}$ & 50,9 & 8,0 \\
\hline
\end{tabular}

específica do $\mathrm{ZrO}_{2}$ e o resultante tamanho médio de partícula não são coerentes, visto que há uma relação inversamente proporcional entre tais grandezas. Tal comportamento é um indício do estado aglomerado do pó. Para $\mathrm{Al}_{2} \mathrm{O}_{3}$ e $\mathrm{SiC}$ o comportamento apresentado entre a área de superfície específica e o correspondente diâmetro médio de partícula pode ser considerado coerente.

\section{Suspensões cerâmicas}

Antes de medir as características eletrocinéticas das suspensões, procedeu-se à avaliação da variação da densidade e viscosidade das soluções alcoólicas de PVB. Tais análises foram realizadas a partir de 5 soluções alcoólicas com diferentes concentrações de PVB. Inicialmente foram realizadas as medidas de densidade das soluções e logo após o respectivo cálculo da viscosidade. Os resultados são exibidos na Tabela II.

Tabela II - Avaliação da densidade e viscosidade das soluções alcoólicas de PVB.

[Table II - Density and viscosity evaluation of alcoholic PVB solutions.]

\begin{tabular}{ccc}
\hline $\begin{array}{c}\text { Concentração } \\
\text { da Solução }(\mathrm{g} / \mathrm{L})\end{array}$ & $\begin{array}{c}\text { Densidade } \\
\left(\mathrm{g} / \mathrm{cm}^{3}\right)\end{array}$ & $\begin{array}{c}\text { Viscosidade }(\eta) \\
\left(\mathrm{mN} . \mathrm{s}^{-2}\right)\end{array}$ \\
\hline 0 & 0,758 & 1,148 \\
0,396 & 0,773 & 1,264 \\
0,784 & 0,780 & 1,284 \\
1,165 & 0,782 & 1,313 \\
1,538 & 0,784 & 1,333 \\
\hline
\end{tabular}

As variações de densidade e viscosidade foram submetidas a interpolação gráfica onde foram obtidas as respectivas funções de ajuste apresentadas na Tabela III. 
Tabela III - Funções de ajuste obtidas a partir de interpolação gráfica.

[Table III - Adjustment functions obtained from graphical interpolation.]

\begin{tabular}{ll} 
Densidade & $\rho=0,0109 x^{3}-0,0399 x^{2}+0,0524 x+0,758$ \\
\hline Viscosidade & $\eta=0,1246 x^{3}-0,3741 x^{2}+0,401 x+1,1496$
\end{tabular}

x: relação entre a massa de PVB adicionada e o volume total da solução.

Suspensões de $\mathrm{Al}_{2} \mathrm{O}_{3}$

As suspensões de $\mathrm{Al}_{2} \mathrm{O}_{3}$ apresentaram uma característica básica em relação ao etanol, com $\mathrm{pa}_{\mathrm{H}}=12,8$ e mobilidade dinâmica de $-0,04 \cdot 10^{-8} \mathrm{~m}^{2} \cdot \mathrm{V}^{-1} \cdot \mathrm{s}^{-1}$. Para este sistema foi medida uma condutividade iônica de $12 \mu \mathrm{S} . \mathrm{cm}^{-1}$ a $25{ }^{\circ} \mathrm{C}$. $\mathrm{Na}$ Fig. 2 observa-se o comportamento das partículas em função do $\mathrm{pa}_{\mathrm{H}}$ na adição de 2 aditivos com características ácidas. A variação de $\mathrm{pa}_{\mathrm{H}}$ compreendida entre 12,8 até 9,8 demonstra que o efeito devido a adsorção do íons $\mathrm{Al}^{3+} \mathrm{e} \mathrm{H}^{+}$é praticamente o mesmo.

O ponto de carga nula, ou seja, a situação para a qual as partículas apresentam mobilidade dinâmica igual a zero ocorre em aproximadamente $\mathrm{pa}_{\mathrm{H}}=11,3$ nas duas situações. O efeito na mobilidade dinâmica para a variação de $\mathrm{pa}_{\mathrm{H}}$ de 9,8 à 4,3 é diferenciado com a adição de $\mathrm{HNO}_{3}$ e $\mathrm{Al}\left(\mathrm{NO}_{3}\right)_{3}$, onde a mobilidade apresenta um aumento bem mais representativo com a adição de $\mathrm{Al}\left(\mathrm{NO}_{3}\right)_{3}$ do que com $\mathrm{HNO}_{3}$. Isto indica que a adsorção específica do íon $\mathrm{Al}^{3+}$ promove um aumento das forças de repulsão partícula/partícula gerando uma melhor dispersão. A carga superficial da partícula anteriormente negativa, passa a ser positiva devido à adsorção destes íons determinantes de potencial.

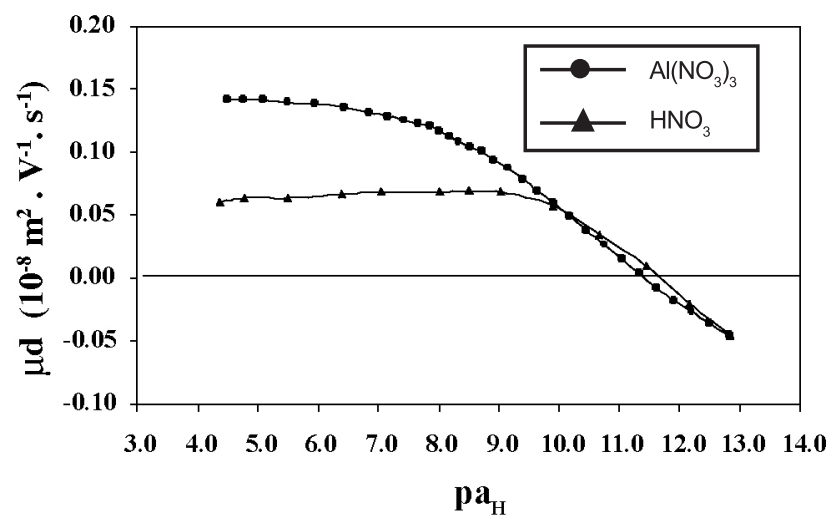

Figura 2: Variação de mobilidade dinâmica em função do $\mathrm{pa}_{\mathrm{H}}$, para a adição das soluções alcoólicas de $\mathrm{HNO}_{3}$ e $\mathrm{Al}\left(\mathrm{NO}_{3}\right)_{3}$.

[Figure 2: Dynamic mobility variation as a function of $p a_{H}$ for the addition of $\mathrm{HNO}_{3}$ and $\mathrm{Al}\left(\mathrm{NO}_{3}\right)_{3}$ alcoholic solutions.]

A Fig. 3 exibe a inversão de carga superficial no $\mathrm{Al}_{2} \mathrm{O}_{3}$ em função do volume injetado da solução de $\mathrm{Al}\left(\mathrm{NO}_{3}\right)_{3}$. Pode ser observado que o ponto isoelétrico ocorre para uma adição de $0,12 \mathrm{~mL}$ de solução, sendo visível também que acima de $0,4 \mathrm{~mL}$ adicionados ocorre uma saturação da adsorção destes íons e o efeito de geração de cargas é muito menos significativo.

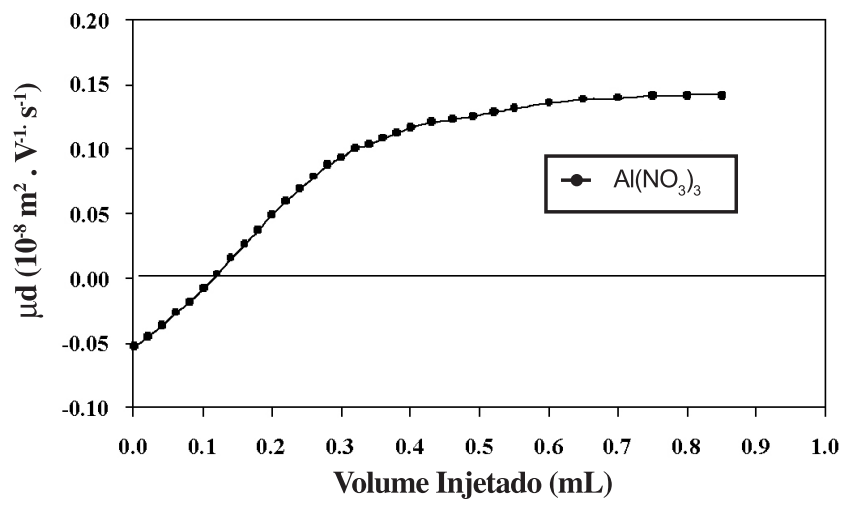

Figura 3: Variação da mobilidade dinâmica em função do volume injetado de solução alcoólica de $\mathrm{Al}\left(\mathrm{NO}_{3}\right)_{3} 0,5 \mathrm{M}$.

[Figure 3: Dynamic mobility variation as a function of the injected alcoholic $\mathrm{Al}\left(\mathrm{NO}_{3}\right)_{3} 0.5 \mathrm{M}$ solution volume.]

O outro aditivo utilizado foi o PVB (normalmente um agente ligante no sistema). Partindo de uma suspensão com $0,4 \mathrm{~mL}$ da solução de $\mathrm{Al}\left(\mathrm{NO}_{3}\right)_{3}$, condição idêntica à da Fig. 3 , foi observada uma diminuição na mobilidade dinâmica das partículas com a adição do PVB. Visto que, de acordo com as análises eletrocinéticas, não ocorreu mudança no valor de $\mathrm{pa}_{\mathrm{H}}$, e que a densidade e a viscosidade do solvente (que variam de acordo com as equações da Tabela III) não influenciam de forma determinante no valor obtido de mobilidade dinâmica, supõe-se que tal diminuição possa ser explicada pela adsorção específica do PVB, que deve alterar o raio hidrodinâmico da partícula diminuindo assim a mobilidade das mesmas.

Outro fator importante nesta suposição da adsorção do PVB, é que para a suspensão em seu estado inicial, ou seja, sem adição de $\mathrm{Al}\left(\mathrm{NO}_{3}\right)_{3}$, não há variação da mobilidade quando o PVB é adicionado, sugerindo ainda

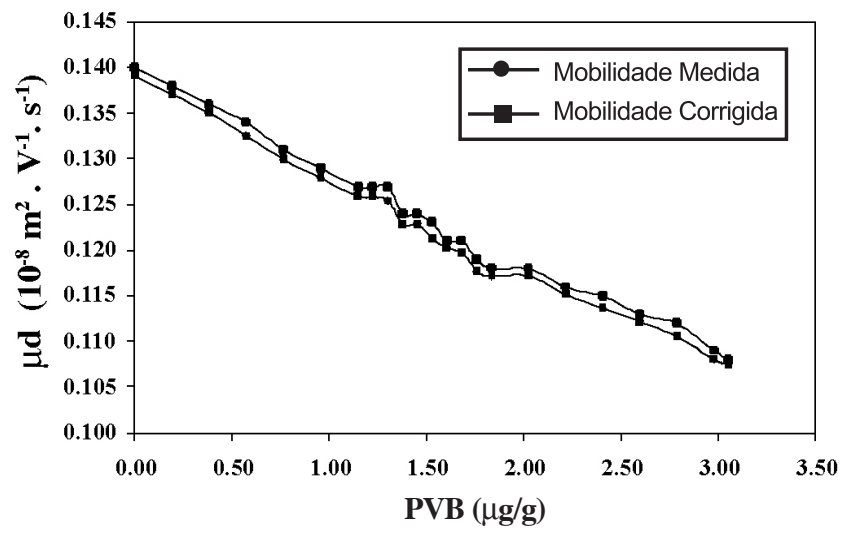

Figura 4: Variação da mobilidade dinâmica em função da massa de PVB adicionada.

[Figure 4: Dynamic mobility as a function of PVB added mass.] 
que a adsorção deste só acontece quando as partículas estão predominantemente carregadas positivamente. A variação da mobilidade dinâmica medida pelo ESA-8000 e a mobilidade corrigida levando em consideração a variação de densidade e viscosidade, pode ser vista na Fig. 4.

\section{Suspensões de SiC}

$\mathrm{O}$ mesmo efeito observado para as suspensões de $\mathrm{Al}_{2} \mathrm{O}_{3}$ também ocorre para as suspensões de $\mathrm{SiC}$. A respectiva caracterização eletrocinética mostra que a superfície das partículas de $\mathrm{SiC}$ possui característica ácida em relação ao etanol, apresentando $\mathrm{pa}_{\mathrm{H}}$ 5,5 e mobilidade dinâmica de $-0,03 \cdot 10^{-8} \mathrm{~m}^{2} \mathrm{~V}^{-1} \cdot \mathrm{s}^{-1}$. Para este sistema, a condutividade iônica medida foi de $4 \mu \mathrm{S} . \mathrm{cm}^{-1}$ a $25^{\circ} \mathrm{C}$. Na Fig. 5, pode ser observado o comportamento da suspensão introduzindo-se 2 aditivos diferentes e com características ácidas; onde demonstrase que o efeito devido à adsorção dos íons $\mathrm{Al}^{3+} \mathrm{e}^{+}$é também diferenciado. Com adição de uma solução de $\mathrm{HCl}$ a mobilidade cai, porém sem atingir o ponto de carga nula, e com a adição $\mathrm{Al}\left(\mathrm{NO}_{3}\right)_{3}$, temos a inversão da carga superficial; igualmente devido à adsorção específica do íon $\mathrm{Al}^{3+}$ Nesta situação ocorre $\mathrm{o}$ aumento da mobilidade dinâmica com a conseqüente melhoria das condições de dispersão das partículas.

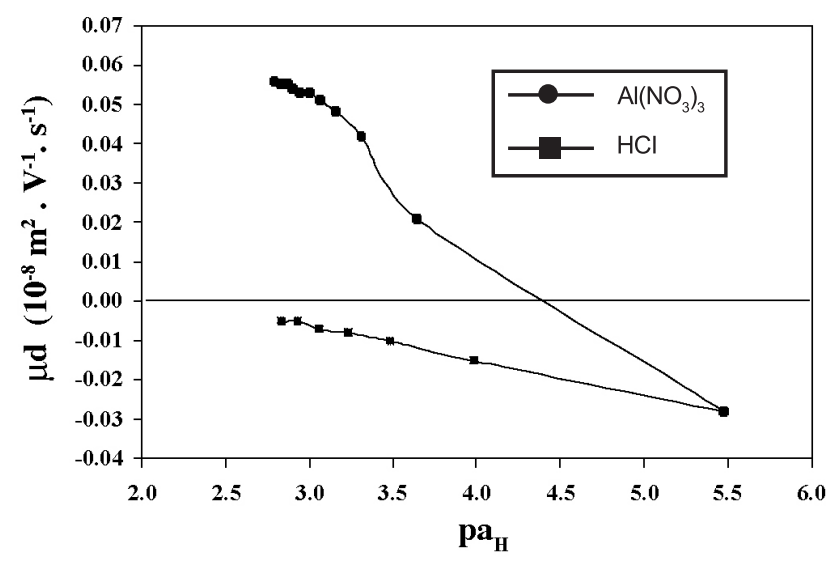

Figura 5: Variação de mobilidade dinâmica em função do $\mathrm{pa}_{\mathrm{H}}$ com adição de soluções alcoólicas de $\mathrm{HCl}$ e $\mathrm{Al}\left(\mathrm{NO}_{3}\right)_{3}$.

[Figure 5: Dynamic mobility variation as a function of $p a_{H}$ with the addition of $\mathrm{HCl}$ and $\mathrm{Al}\left(\mathrm{NO}_{3}\right)_{3}$ alcoholic solutions.]

A Fig. 6 exibe a inversão de carga superficial no $\mathrm{SiC}$ em função do volume de $\mathrm{Al}\left(\mathrm{NO}_{3}\right)_{3}$ injetado. Observa-se que com $0,025 \mathrm{~mL}$ de solução adicionada, a carga superficial das partículas é nula; e que com $0,2 \mathrm{~mL}$ da solução ocorre uma saturação da adsorção destes íons. A partir deste volume não ocorre mais o efeito de geração de cargas.

Partindo-se, novamente, de uma suspensão de $\mathrm{SiC}$ com 0,2 $\mathrm{mL}$ da solução de $\mathrm{Al}\left(\mathrm{NO}_{3}\right)_{3}$, mesma situação mostrada na Fig. 6, com a adição da solução de PVB foi observada também uma diminuição da mobilidade, porém não tão intensa como no caso da alumina, onde com uma quantidade 20 vezes menor de PVB a diminuição da mobilidade foi 3 vezes maior em relação ao

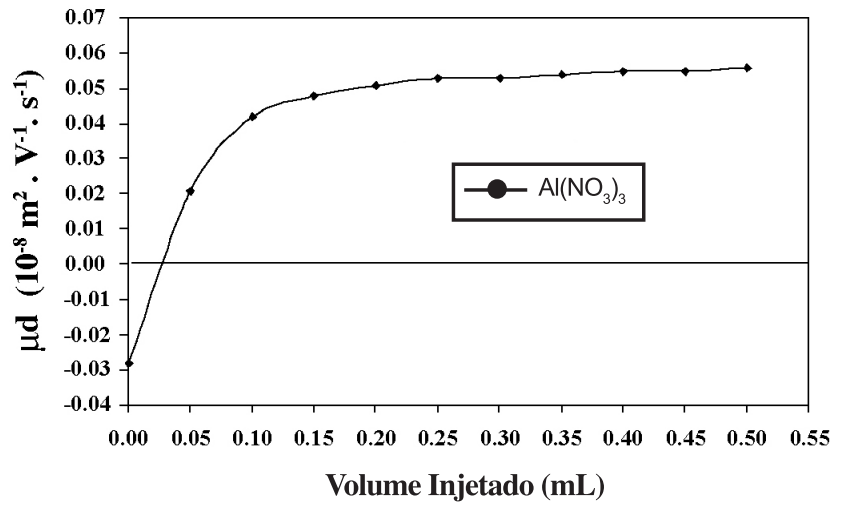

Figura 6: Variação da Mobilidade dinâmica em função do volume injetado de solução alcoólica de $\mathrm{Al}\left(\mathrm{NO}_{3}\right)_{3}$ 0,5 M.

[Figure 6: Dynamic mobility variation as a function of the injected alcoholic $\mathrm{Al}\left(\mathrm{NO}_{3}\right)_{3} \mathrm{O} .5 \mathrm{M}$ solution volume.]

SiC. Esta diferença provavelmente se deve à diferença de área de superfície específica dos materiais, que no caso do $\mathrm{Al}_{2} \mathrm{O}_{3}$ tem a relação de 2,4 para 1 em relação ao SiC. Visto que o diâmetro equivalente da partícula de SiC é bem maior que o da partícula de alumina, e a molécula adsorvida é a mesma, logo, o efeito deve ser maior na partícula menor. A variação da mobilidade dinâmica medida pelo ESA-8000 e a mobilidade corrigida considerando a variação de densidade e viscosidade é apresentada na Fig. 7.

Com o intuito de novamente confirmar uma suposta adsorção do PVB que se dá preferencialmente em superfície positiva, foi preparada uma suspensão, sem adição de $\mathrm{Al}\left(\mathrm{NO}_{3}\right)_{3}$, e foi verificado que também não há variação da mobilidade quando o PVB é adicionado, confirmando que a adsorção deste, só acontece quando as partículas estão predominantemente carregadas positivamente.

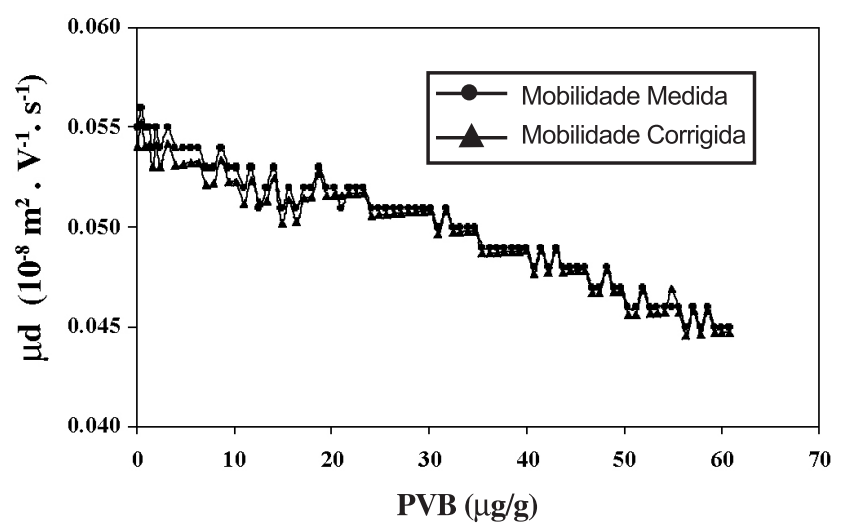

Figura 7: Variação da mobilidade dinâmica em função da massa adicionada de PVB.

[Figure 7: Dynamic mobility variation as a function of the PVB added mass.]

\section{Suspensões de $\mathrm{ZrO}_{2}-\mathrm{MgO}$}

Diferentemente das suspensões de $\mathrm{Al}_{2} \mathrm{O}_{3}$ e de $\mathrm{SiC}$, isoladamente, o sistema $\mathrm{ZrO}_{2}-\mathrm{MgO}$ apresenta-se como uma 
combinação de um óxido ácido em relação ao EtOH $\left(\mathrm{ZrO}_{2}\right)$ com um óxido básico $(\mathrm{MgO})$, também em relação ao EtOH. Mesmo estando numa concentração menor ( $3 \%$ em peo), o $\mathrm{MgO}$ determina a carga elétrica superficial do sistema que é predominantemente positiva. A caracterização deste comportamento e as suas conseqüências para o processo de estabilização eletrocinética já foram apresentadas e discutidas em trabalho anterior [18]. O que se observa é que não ocorre simplesmente uma neutralização dos sítios ácidos na superfície do $\mathrm{ZrO}_{2}$, mas sim um carregamento intenso da superfície, caracterizado pela presença de cargas positivas.

A caracterização eletrocinética da suspensão mostra que o conjunto de partículas de $\mathrm{ZrO}_{2}-\mathrm{MgO}$ possui característica básica em relação ao etanol, apresentando $\mathrm{pa}_{\mathrm{H}}=9,0 \mathrm{e}$ mobilidade dinâmica de $0,03 \cdot 10^{-8} \mathrm{~m}^{2} \mathrm{~V}^{-1} \cdot \mathrm{s}^{-1}$. Para este sistema, a condutividade iônica medida foi de $26 \mu \mathrm{S} . \mathrm{cm}^{-1}$ a $25^{\circ} \mathrm{C}$.

A esta suspensão foram adicionadas quantidades controladas de uma solução alcoólica de PVB com concentração de $30 \mathrm{~g} / \mathrm{L}$ e a respectiva caracterização eletrocinética apresentada na Fig. 8.

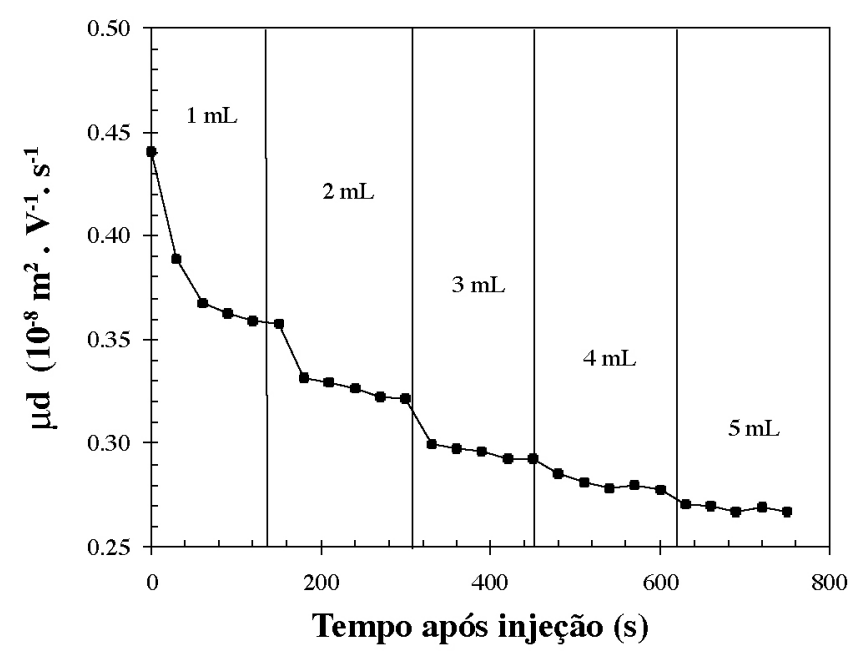

Figura 8: Comportamento eletrocinético como uma função da quantidade de aditivo e do tempo para o sistema $\mathrm{ZrO}_{2}-\mathrm{MgO}$.

[Figure 8: Electrokinetic behaviour as a function of additive amount and time for the $\mathrm{ZrO}_{2}-\mathrm{MgO}$ system.]

Nesta caracterização foi avaliada não apenas a quantidade de aditivo injetado, mas também a variação da mobilidade em função do tempo. Desta maneira, pode-se avaliar a adsorção das moléculas poliméricas de um ponto de vista cinético. Observa-se que a injeção da solução de PVB promove, unicamente, a diminuição da intensidade das cargas elétricas, o que é refletido na diminuição de mobilidade dinâmica. Esta diminuição ocorre em tempos extremamente curtos indicando que a interação das moléculas poliméricas com a superfície das partículas é instantânea.

A análise destes dados, sob outro ponto de vista, permite estabelecer que a possibilidade da diminuição da mobilidade causada por um efeito de compressão de dupla camada elétrica é mínima. Primeiro porque o PVB não se torna ionizado quando solubilizado no etanol devido à baixa constante dielétrica deste último. Em segundo lugar, não estando ionizado não há como promover um incremento na força iônica da suspensão, acarretando ao efeito de compressão da dupla camada elétrica.

Possivelmente a adsorção do PVB deve ocorrer por pontes de hidrogênio e não por atração eletrostática, e a sua adsorção deve promover, a exemplo do que já foi sugerido para as suspensões de $\mathrm{Al}_{2} \mathrm{O}_{3}$ e de $\mathrm{SiC}$, um aumento do raio hidrodinâmico das partículas que tem influência direta na mobilidade das partículas.

A Fig. 9 exibe a variação de $\mathrm{pa}_{\mathrm{H}}$ em função do tempo de injeção das alíquotas da solução alcoólica de PVB. É nítida a forte variação nos valores de $\mathrm{pa}_{\mathrm{H}}$ que ocorre nos instantes iniciais após a injeção da solução de PVB, diferentemente das suspensões de $\mathrm{Al}_{2} \mathrm{O}_{3}$ e de $\mathrm{SiC}$, onde não houve variação. Nota-se também que o sistema tende a voltar ao valor inicial de $\mathrm{pa}_{\mathrm{H}}$, o que permite presumir que se trata de uma situação de equilíbrio. Considerando então a variação de $\mathrm{pa}_{\mathrm{H}}$ neste sistema, sugerimos que deva ter ocorrido uma competição entre o PVB e o íon etóxido, resultante de reação superficial entre as partículas cerâmicas e o etanol, conforme referência [18], que não ocorre para $\mathrm{o}_{2} \mathrm{Ol}_{3}$ e o SiC.

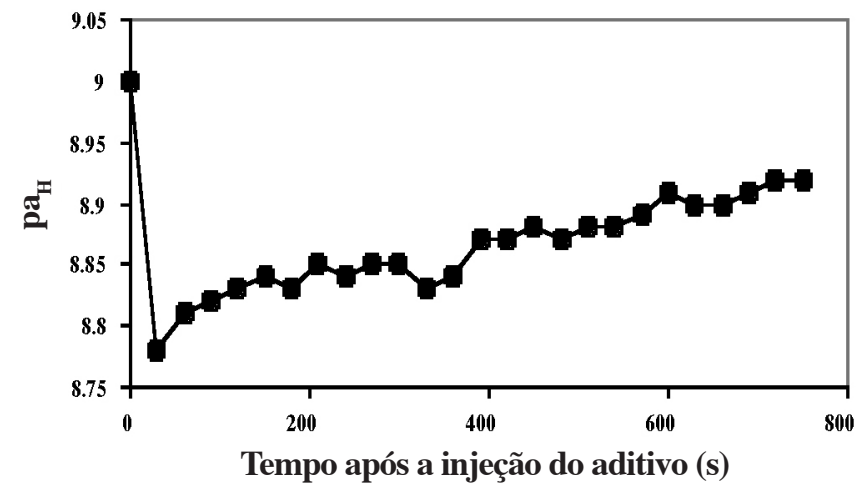

Figura 9: Variação do $\mathrm{pa}_{\mathrm{H}}$ em função do tempo para o sistema $\mathrm{ZrO}_{2}$ $\mathrm{MgO}$.

[Figure 9: $\mathrm{pa}_{\mathrm{H}}$ variation as a function of time for the $\mathrm{ZrO}_{2}-\mathrm{MgO}$ system.]

\section{CONCLUSÕES}

A caracterização dos óxidos utilizada indica que não deve haver problemas no processamento dos mesmos devido às características físicas, pois os resultados mostraram que os pós são de baixa granulometria e área de superfície específica considerada satisfatória para processamento de suspensões; apenas uma ressalva para o estado de aglomeração em que o $\mathrm{ZrO}_{2}$ se encontra, que pode ser resolvido relativamente fácil com ultra-som ou moagem.

As análises eletrocinéticas mostraram que $\mathrm{o} \quad \mathrm{Al}_{2} \mathrm{O}_{3}$ tem características básicas e o $\mathrm{SiC}$ características ácidas em relação ao etanol, porém nos dois casos o sistema se 
encontra com as partículas carregadas predominantemente com cargas negativas. Diferentemente do $\mathrm{ZrO}_{2}-\mathrm{MgO}$ que as partículas se encontram carregadas predominantemente com cargas positivas.

Adicionando o aditivo PVB, pôde-se observar que o mesmo adsorve (através de variação na mobilidade dinâmica das partículas) apenas em partículas com superfície carregadas com cargas positivas que é o caso do sistema $\mathrm{ZrO}_{2}-\mathrm{MgO}$, este indício fica reforçado a partir dos resultados obtidos com as suspensões de $\mathrm{Al}_{2} \mathrm{O}_{3}$ e $\mathrm{SiC}$ que inicialmente quando adicionados em etanol estão com cargas superficiais negativas, neste caso não a variação de mobilidade quando adicionado o PVB indicando que não há adsorção.

No entanto, quando é inserido através de uma solução de $\mathrm{Al}\left(\mathrm{NO}_{3}\right)_{3}$ os íons $\mathrm{Al}^{3+}$ na suspensão promovem a geração de cargas positivas nas partículas por adsorção específica mostrando que quando adicionamos o PVB ocorre variação na mobilidade dinâmica das partículas confirmando a adsorção.

\section{AGRADECIMENTOS}

À Alcoa Alumínio S. A. e ao IPEN pelo fornecimento das matérias-primas e à FAPESP (processos 99/10798-0 e 99/03375-6) pelo apoio financeiro a esta pesquisa.

\section{REFERÊNCIAS}

[1] P. C. Hiemenz; Principles of Colloid and Surface Chemistry, $2^{\text {nd }} E d$. Marcel Dekker, New York (1986) p. 815.

[2] F. S. Ortega, V. C. Pandolfelli, J. A. Rodrigues, D. P. F. Souza, Cerâmica 43, 280 (1997) 77.

[3] B. V. Derjaguin, L. Landau, Acta Physicochim. 14 (1941) 633.

[4] I. R. Oliveira, A. R. Studart, R. G. Pillegi, V.C. Pandolfelli, Dispersão e Empacotamento de Partículas, Fazendo Arte Editorial, S. Paulo (2000) p. 12.

[5] E. J. W. Verwey, J. T. G. Overbeek, Theory of the Stability of Lyophobic Colloids, Elsevier Publ. Co. Inc., Amsterdam (1948) pp. 1-63.

[6] W. M. Sigmund, N. S. Bell, L. Bergström, J. Am. Ceram. Soc. 83, 7 (2000) 1557.

[7] H. N. Stein, Rheological Behavior of Suspensions, in N. P. Cheremisinoff (Ed.), Encyclopedia of Fluid Mechanics: Slurry Flow Techology, Gulf Publishing, Houston 5 (1986) pp. 3-47.

[8] J. S. Reed, Principles of Ceramics Processing, $2^{\text {nd }}$ Ed., John Wiley \& Sons, New York (1995) p. 273.

[9] T. Hotta, H. Abe, M. Naito, M. Takahashi, K. Uematsu, Z. Kato, Powder Technology 149, 2-3 (2005) 106.

[10] Y. Takao, T. Hotta, M. Naito, N. Shinohara, M. Okumiya, K. Uematsu, J. Eur. Ceram. Soc. 22, 4 (2002) 397.

[11] H. Koelmans, J. Th. G. Overbeek, Discuss. Faraday
Soc. 18 (1954) 52.

[12] J. Mizuguchi, K. Sumi, T. Muchi, J. Electrochem. Soc. 130 (1983) 1819.

[13] S. Ananthakumar, K. Prabhakaran, U. S. Hareesh, P. Manohar, K. G. K. Warrier, Mater. Chem. Phys. 85, 1 (2004) 151.

[14] K. Prabhakaran, S. Ananthakumar, C. Pavithran, J. Eur. Ceram. Soc. 19, 16 (1999) 2875.

[15] P. Sarkar, P. S. Nicholson, J. Am. Ceram. Soc. 79, 8 (1996) 1987.

[16] D. Gouvêa, B. B. S. Murad, Cerâmica 45, 292-293 (1999) 103.

[17] R. Moreno, B. Ferrari, Am. Ceram. Soc. Bull. 79, 1 (2000) 44.

[18] P. J. B. Marcos, R. H. R. Castro, D. Gouvêa, Cerâmica 47, 304 (2001) 187.

[19] J. Widegren, L. Bergström, J. Am. Ceram. Soc. 85, 3 (2002) 523.

[20] J. Widegren, L. Bergströn, J. Eur. Ceram. Soc 20 (2000) p. 659.

[21] P. H. C. Van der Hoeven, J. Lyklema, Adv. Coll. Sci. 42 (1992) 205.

[22] G. Wang, P. Sarkar, P. S. Nicholson, J. Am. Ceram. Soc. 80, 4 (1997) 965.

[23] M. D. Chadwick, J. W. Goodwin, E. J. Lawson. P. D. A. Mills, B. Vincent, Colloids and Surfaces A: Physicochem. Eng. Aspects 203 (2002) 229.

[24] D. J. Shanefield, Organic Additives and Ceramic Processing with Applications in Powder Metallurgy, Ink, and Paint, Kluwer Academic Publishers, Boston (1995) p. 236.

[25] R. W. O'Brien, Determination of Particle Properties from Electroacoustic Measurements, in Handbook on Characterization Techniques for the Solid-solution Interface, Eds. J. H. Adair, J. A. Casey, S. Venigalla, The Am. Ceram. Soc. Inc., Ohio (1993) p. 113.

[26] E. C. Subbarao, in Advances in Ceramics, Vol. 3. Science and Technology of Zirconia, The Am. Ceram. Soc. Inc., Ohio (1981) p. 8.

[27] R. Stevens, Zirconia and Zirconia Ceramics, Magnesium Elektron Ltd., UK (1986) p. 39.

[28] P. J. B. Marcos, Dissertação de Mestrado, EPUSP, S. Paulo (2001).

[29] Y. Hirata, K. Miyano, S. Sameshima, Y. Kamino, Colloids and Surfaces A: Physicochem. Eng. Aspects 133, 3 (1998) 183.

[30] D. J. Shaw, Introdução à Química dos Colóides e de Superfícies, Ed. Edgard Blücher Ltda., S. Paulo (1975) p. 145.

[31] Matec Applied Sciences, ESA-8000 System Operating Manual, Matec Applied Sciences Inc., Massachussets (1998).

[32] A. Borner, R. Herbig, Colloids and Surfaces A: Physicochem. Eng. Aspects 159, 2-3 (1999) 439.

(Rec. 04/09/2006, Ac. 24/11/2006) 